Proyecciones

Vol. $18, N^{\circ} 1$, pp. 77-89, July 1999

Universidad Católica del Norte

Antofagasta - Chile

\title{
THE DUAL BADE THEOREM IN LOCALLY CONVEX SPACES AND REFLEXIVITY OF A CLOSED UNITAL SUBALGEBRA
}

\author{
ÖMER GÖK \\ Yildiz Technical University, Istanbul - TURKEY
}

\begin{abstract}
The results presented in this paper extend a dual version of the reflexivity theorem of $W$. Bade to locally convex spaces. Dual version of the Bade theorem in a Banach $C(K)$-module was firstly discovered in [1]. It is our aim to extend it to a locally convex $C(K)$-module. As a consequence, it is proven that each unital $w^{*}$ operator topology closed subalgebra of the $w^{*}$ operator topology closed algebra generated by a Boolean algebra of projections is reflexive.
\end{abstract}

1991 Mathematics Subject Classification.

Primary : 46H99, 47030 .

Key words and phrases : reflexivity, Boolean algebra, quasicomplete. 


\section{Introduction}

In this article, we investigate the role played by lattice order, locally convex $C(K)$-module and $f$-module in the study of operator algebras generated by Boolean algebras of projections. The study of operator algebras generated by Bade complete ( $\sigma$-complete) Boolean algebras of projections in Banach spaces was firstly given by W. G. Bade in [5]. The reflexivity result of Bade asserts that a continuous linear operator $T$ on a Banach space $X$ belongs tothe strongly closed algebra generated by a $\sigma$-complete Boolean algebra $B$ of projections if and only if $T$ leaves invariant each $B$-invariant (closed) subspace of $X$. Furthermore, this idea developed by T. A. Gillespie, [10], and C. Rall, [12].

The reflexivity theorem of Bade by a systematic approach to the locally convex spaces was extended in [6], [7], [8], [13] and [14]. The reflexive operator algebras in a Banach $C(K)$-module were studied in [11] and [1].

\section{Preliminaries}

Let $C(K)$ be the set of all continuous real or complex valued functions with the suprenum norm on a compact Hausdorff space $K$. Suppose that $X$ is a locally convex Hausdorff space. We call that $X$ is a locally convex $C(K)$-module if the bilinear mapping

$$
C(K) \times X \rightarrow X
$$

satisfies the following conditions:

(i) $1 . \mathrm{x}=\mathrm{x}$ for all $x \in X, \quad 1 \in C(K)$,

(ii) (ab).x=a.(b.x) for all $a, b \in C(K), \quad x \in X$,

(iii) The bilinear mapping (1) is separately continuous.

We let $X^{\prime}$ denote the continuous dual of a locally convex Hausdorff space $X$, let $X^{\prime \prime}$ denote the second continuous dual of a locally convex Hausdorff space $X$ and let $X^{*}$ denote an algebraic dual of a locally convex Hausdorff space $X$. Let $X$ be a quasicomplete locally convex Hausdorff space and let $B$ be an equicontinuous Boolean algebra in the sequentially complete space. By $\mathcal{L}(X)$ we denote the space of all continuous linear operators from $X$ into itself equipped with the topology of pointwise convergence in $X$. A Boolean algebra of projections in $\mathcal{L}(X)$ is a family $B$ of commuting idempotents, 
partially ordered with respect to range inclusion, which is a Boolean algebra with respect to the lattice operations given by $E \vee F=E+F-E F$ and $E \wedge F=E F$ for all $E, F \in B$. We assume that the unit element of $B$ is $I$. We say that an equicontinuous Boolean algebra of projections $B$ in the locally convex space $B$ is strongly equicontinuous if $E_{n}$ converges to 0 strongly for any disjoint sequence $\left\{E_{n}\right\}$ in $B$. It will always be assumed that $X$ is quasicomplete and $\mathcal{L}(X)$ is sequentially complete. In this work all Riesz spaces will be assumed as Archimedean. For the terminology, notations and elementary properties of Riesz spaces, elementary f-algebra theory and orthomorphism not explained or proved in this paper we refer to [2], [3] and [15].

Suppose that $K$ is the Stone space of $B$. Let $Q(K)$ be the set of all open and closed sets in $K$ and let $m_{1}$ be the Boolean isomorphism from $Q(K)$ onto $B$. We denote by $A$ the linear span of $B$ and by $H$ the linear span of $Q(K)$. As shown in [6], the closed algebra $\bar{A}$ is complete. The linear mapping $m$ from $H$ to $\bar{A}$ is continuous, $m(1)=I$; so we can extend it, say $m$, from $C(K)$ into $\bar{A}$. It is well-known that $C(K)$ is $|\sigma|\left(C(K)^{\prime \prime}, C(K)^{\prime}\right)$ -dense in $C(K)^{\prime \prime},[3]$ Theorem 11.16.

Then the bilinear mapping

$$
C(K) \times X \rightarrow X
$$

is separately continuous. On $X^{\prime}$ we put $\sigma\left(X^{\prime}, X\right)$, on $C(K)^{\prime}$ we consider $\sigma\left(C(K)^{\prime}, C(K)^{\prime \prime}\right)$-topology.

Hence we can establish the following bilinear mappings in three steps:

$$
\begin{gathered}
X \times X^{\prime} \rightarrow C(K)^{\prime} ;\left(x, x^{\prime}\right) \rightarrow\left(x \cdot x^{\prime}\right)(a)=x^{\prime}(a . x), \\
C(K)^{\prime \prime} \times X^{\prime} \rightarrow X^{\prime} ;\left(a, x^{\prime}\right) \rightarrow\left(a \cdot x^{\prime}\right)(x)=a\left(x . x^{\prime}\right), \\
C(K)^{\prime \prime} \times X^{\prime *} \rightarrow X^{\prime *} ;(a, \gamma) \rightarrow(a \cdot \gamma)\left(x^{\prime}\right)=\gamma\left(a . x^{\prime}\right) .
\end{gathered}
$$

We need to put a locally convex $C(K)^{\prime \prime}$-module structure on $X^{\prime}$. To do this, it is our aim to prove that the bilinear mapping (3) is separately continuous.

We fix $a \in C(K)^{\prime \prime}$ and $x^{\prime} \in X^{\prime}$. For if $\left\{x_{\alpha}\right\}$ is a net in $X$ which converges to $x$, then by the scparate continuity of the bilinear mapping (2) $x_{\alpha} \cdot x^{\prime} \rightarrow x \cdot x^{\prime}$ in $C(K)^{\prime} . a\left(x_{\alpha} \cdot x^{\prime}\right) \rightarrow a\left(x \cdot x^{\prime}\right)$ for each $a \in C(K)^{\prime \prime}$, i.e. , $\left(a . x^{\prime}\right)\left(x_{\alpha}\right) \rightarrow\left(a \cdot x^{\prime}\right) x$. Hence the bilinear mapping (3) is well-defined. To 
prove the continuity of the bilinear mapping (3) in the first coordinate, we fix $x^{\prime} \in X^{\prime}$. Let $\left\{a_{\alpha}\right\}$ be a net in $C(K)^{\prime \prime}$ which converges to $a$ with respect to $\sigma\left(C(K)^{\prime \prime}, C(K)^{\prime}\right)$-topology. For $x \in X$ and $x^{\prime} \in X^{\prime}$, $x \cdot x^{\prime} \in C(K)^{\prime}$ by $(2)$. Therefore, $a_{\alpha}\left(x \cdot x^{\prime}\right) \rightarrow a\left(x . x^{\prime}\right)$, i.e., $a_{\alpha} \cdot x^{\prime} \rightarrow a \cdot x^{\prime}$. To prove the continuity of the bilinear mapping (3) in the second coordinate, we fix $a \in C(K)^{\prime \prime}$. Let $\left\{x_{\alpha}^{\prime}\right\}$ be a net in $X^{\prime}$ which converges to $x^{\prime}$ with respect to $\sigma\left(X^{\prime}, X\right)$. By the bilinear mapping (2) $x . x_{\alpha}^{\prime}$ converges to $x \cdot x^{\prime}$ in $C(K)^{\prime}$. Therefore, $a\left(x \cdot x_{\alpha}^{\prime}\right)$ converges to $a\left(x . x^{\prime}\right)$, i.e., $a . x_{\alpha}^{\prime} \rightarrow a \cdot x^{\prime}$. For more information the bilinear mapping (3) we refer to Lemma 1.

The mapping (4) is only a bilinear mapping. Let $X$ be a locally convex $C(K)$-module and $x \in X$. Then the set $\Delta(x)$ is defined by

$$
\Delta(x)=\{a \cdot x:\|a\| \leq 1, \quad a \in C(K)\}^{-},
$$

where closure is taken with respect to the given topology in $X$. Similarly, by bilinear mapping (3) for $x^{\prime} \in X^{\prime}$ the set $\Delta\left(x^{\prime}\right)$ is defined by

$$
\Delta\left(x^{\prime}\right)=\left\{a \cdot x^{\prime}:\|a\| \leq 1, \quad a \in C(K)^{\prime \prime}\right\}^{-},
$$

where closure is taken with respect to the $\sigma\left(X^{\prime}, X\right)$ topology on $X^{\prime}$. A linear subspace $Y$ of a locally convex $C(K)$-module $X$ is called an ideal if for each $x \in Y \Delta(x) \in Y$. Similarly, an ideal for a lincar subspace of a locally convex $C(K)^{\prime \prime}$-module $X^{\prime}$ can be defined. Let $Y$ bo an ideal in a locally convex $C(K)$-module $X$. We say that center of $Y$ denoted by $Z_{m}(Y)$ is the set of all linear operators $T$ from $Y$ to $Y$ such that $T y \in \lambda \Delta(y)$ for each $y \in Y$, where $\lambda=\lambda(T)$ is a positive number, i. e.,

$$
Z_{m}(Y)=\{T: Y \rightarrow Y \mid(\exists \lambda \geq 0) \quad(\forall y \in Y) \quad(T y \in \lambda \Delta(y))\} .
$$

Similarly, the center of $Y$ for an ideal $Y$ in a locally convex $C(K)^{\prime \prime}$ - module $X^{\prime}$ can be defined. Similar definitions can be found in [1].

If the bilinear mapping (1) is taken as $X=C(K)$, then the bilinear mapping (4) defined by $C(K)^{\prime \prime} \times C(K)^{\prime \prime} \rightarrow C(K)^{\prime \prime},(a, b) \rightarrow(a, b)(c)=$ $b(a . c)$ is continuous and defines the Arens nultiplication on $C(K)^{\prime \prime}$ and $C(K)^{\prime \prime}$ is isomorphic to $C(S)$ with $S$ Hyperstonian. Proof of the following Lemma was given in [9].

\section{Lemma 1}

Let $X$ be a quasicomplete locally convex Hausdorff space such that $\mathcal{L}(X)$ is sequentially complete and let $B \subseteq \mathcal{L}(X)$ be an equicontinuous Boolean algebra of projections. Then the following statements are truc. 
(i) The bilinear mapping (1) defines a locally convex $C(K)$-module on $X$.

(ii) The bilinear mapping (3) defines a locally convex $C(S)$-module on $X^{\prime}$ that gives a homomorphism $m^{*}: C(K)^{\prime \prime} \rightarrow \mathcal{L}\left(X^{\prime}\right)$ defined by $m^{*}(a)\left(x^{\prime}\right)=a x^{\prime}$.

(iii) For each $a \in C(K), m^{*}(a)=(m(a))^{*}$, where $(m(a))^{*}$ is the adjoint of $m(a)$.

(iv) $m(1)=I$.

(v) $m^{*}(1)=I^{\prime}$, where $I^{\prime}$ is the adjoint of $I$.

(vi) $m^{*}$ is $\left(w^{*}, w^{*}\right.$-operator $)$ continuous.

\section{Proof. (ii)}

$(a+b) \cdot x^{\prime}=a \cdot x^{\prime}+b \cdot x^{\prime}$ for $a, b \in C(K)^{\prime \prime}, \quad x^{\prime} \in X^{\prime}$. For proof of this condition, let $x \in X$ be arbitrary, then

$$
\begin{gathered}
(a+b) \cdot x^{\prime}(x)=(a+b)\left(x \cdot x^{\prime}\right)=a\left(x \cdot x^{\prime}\right)+b\left(x \cdot x^{\prime}\right) \\
=\left(a \cdot x^{\prime}\right)(x)+\left(b \cdot x^{\prime}\right)(x)=\left(a \cdot x^{\prime}+b \cdot x^{\prime}\right)(x) .
\end{gathered}
$$

As $x$ is arbitrary we conclude that $(a+b) \cdot x^{\prime}=a \cdot x^{\prime}+b \cdot x^{\prime}$.

$a .\left(x^{\prime}+y^{\prime}\right)=a \cdot x^{\prime}+a \cdot y^{\prime}$ for $a \in C(K)^{\prime \prime}, \quad x^{\prime}, y^{\prime} \in X^{\prime}$. For proof of this claim, let $x \in X$ be arbitrary, then

$$
\begin{array}{r}
\left(a \cdot\left(x^{\prime}+y^{\prime}\right)\right)(x)=a\left(x \cdot\left(x^{\prime}+y^{\prime}\right)\right)=a\left(x \cdot x^{\prime}+x \cdot y^{\prime}\right)=a\left(x \cdot x^{\prime}\right)+a\left(x \cdot y^{\prime}\right)= \\
=\left(a \cdot x^{\prime}+a \cdot y^{\prime}\right)(x) .
\end{array}
$$

As $x$ is arbitrary we conclude that $a \cdot\left(x^{\prime}+y^{\prime}\right)=a \cdot x^{\prime}+a \cdot y^{\prime}$. Therefore, (3) is a bilinear mapping. $x^{\prime} .1=x^{\prime}$ for all $x^{\prime} \in X^{\prime}$. For this, let $x \in X$, then

$$
\begin{gathered}
\left(x^{\prime} .1\right)(x)=1\left(x \cdot x^{\prime}\right)=\left(x \cdot x^{\prime}\right)(1) \\
=x^{\prime}(1 . x)=x^{\prime}(x) .
\end{gathered}
$$

As $x$ is arbitrary, hence condition is satisfied.

$(a . b) \cdot x^{\prime}=a \cdot\left(b \cdot x^{\prime}\right)$ for $a, b \in C(K)^{\prime \prime}, x^{\prime} \in X^{\prime}$. To prove this condition let $x \in X$, then

$$
(a \cdot b) \cdot x^{\prime}(x)=(a \cdot b)\left(x \cdot x^{\prime}\right)=a\left(b \cdot\left(x \cdot x^{\prime}\right)\right)
$$


On the other hand, by the definition of the bilinear mapping (3) b. $x^{\prime} \in X^{\prime}$ and

$$
a .\left(b \cdot x^{\prime}\right)(x)=a\left(\left(b \cdot x^{\prime}\right) \cdot x\right)
$$

Hence, it suffices to show that

$$
b \cdot\left(x \cdot x^{\prime}\right)=\left(b \cdot x^{\prime}\right) \cdot x
$$

for cach $x \in X, x^{\prime} \in X^{\prime}$ and $b \in C(K)^{\prime \prime}$. To this end, let $a \in C(K)$. Then

$$
\left(b \cdot\left(x \cdot x^{\prime}\right)\right)(c)=b\left(c \cdot\left(x \cdot x^{\prime}\right)\right)
$$

by the definition of bilinear mapping (3). On the other hand,

$$
\left(x \cdot\left(x^{\prime} \cdot b\right)\right)(c)=\left(x^{\prime} \cdot b\right)(c \cdot x)=b\left((c . x) \cdot x^{\prime}\right)
$$

by the definitions of bilinear mapping (2) and (3). Thercfore, it is enough to show that $c .\left(x \cdot x^{\prime}\right)=(c . x) \cdot x^{\prime}$ for each $c \in C(K), x \in X, x^{\prime} \in X^{\prime}$. Let $d \in C(K)$ be arbitrary, then

$$
\left(c .\left(x \cdot x^{\prime}\right)\right)(d)=\left(x \cdot x^{\prime}\right)(\text { d.c })=x^{\prime}((d . c) x) .
$$

Calculating the right hand side,

$$
\left((c . x) \cdot x^{\prime}\right)(d)=x^{\prime}(d(c . x))=x^{\prime}((d . c) x) .
$$

Therefore, $(a \cdot b) \cdot x^{\prime}=a \cdot\left(b \cdot x^{\prime}\right)$. It remains to prove the bilinear mapping (3) is separately continuous. The continuity of the bilinear mapping (3) in the second coordinate from $\left(X^{\prime}, \sigma\left(X^{\prime}, X\right)\right)$ into $\left(X^{\prime}, \sigma\left(X^{\prime}, X\right)\right)$ is an immediate consequence of the following paragraph.

Let us equip $X^{\prime}$ with $\sigma\left(X^{\prime}, X\right), C(K)^{\prime \prime}$ with the norm topology and consider the space $X^{\prime} \times C(K)^{\prime \prime}$ with the induced product topology. We claim the bilinear mapping (3) is continuous when the domain is equipped with the product topology and the range is equipped witl $\sigma\left(X^{\prime}, X\right)$. Let $\left\{y_{\alpha}\right\}=\left\{\left(a_{\alpha}, x_{\alpha}^{\prime}\right)\right\}$ be a net in $C(K)^{\prime \prime} \times X^{\prime}$ convergent to zero in the product topology. Let $x \in X$ be arbitrary, we have

$$
\left|\left(y_{\alpha}\right)(x)\right|=\left|\left(a_{\alpha} \cdot x_{\alpha}^{\prime}\right)(x)\right|=\left|a_{\alpha}\left(x \cdot x_{\alpha}^{\prime}\right)\right| \leq\left\|a_{\alpha}\right\|\left\|x \cdot x_{\alpha}^{\prime}\right\|
$$

As the bilinear mapping (2) is continuous from $\left(X^{\prime}, \sigma\left(X^{\prime}, X\right)\right)$ into $\left(C(K)^{\prime}\right.$, $\sigma\left(C(K)^{\prime}, C(K)\right)$, we have $\left(x \cdot x_{\alpha}^{\prime}\right)$ converges to zero in $\sigma\left(C(K)^{\prime}, C(K)\right.$. An application of the Uniform Boundedness Principle shows that the $\left\{x . x_{\alpha}^{\prime}\right\}$ is 
bounded in the norm topology of $C(K)^{\prime}$. As $x \in X$ is arbitrary, this yields that $\left\{y_{\alpha}\right\}$ converges to zero in $X^{\prime}$ with respect to $\sigma\left(X^{\prime}, X\right)$.

To prove the continuity of the bilinear mapping (3) in the first coordinate, we let $\left\{a_{\alpha}\right\}$ be a net in $C(K)^{\prime \prime}$ which converges to $a$ in $\sigma\left(C(K)^{\prime \prime}, C(K)^{\prime}\right)$. Let $x \in X, \quad x^{\prime} \in X^{\prime}$ be fixed but arbitrary elements. As $x \cdot x^{\prime} \in C(K)^{\prime}$ we have

$$
\lim _{\alpha}\left(a_{\alpha} \cdot x^{\prime}\right)(x)=\lim _{\alpha} a_{\alpha}\left(x \cdot x^{\prime}\right)=a\left(x \cdot x^{\prime}\right)=\left(a \cdot x^{\prime}\right)(x) .
$$

As $x$ is arbitrary, we conclude that the bilinear mapping (3) is continuous from $\sigma\left(C(K)^{\prime \prime}, C(K)^{\prime}\right)$ into $\sigma\left(X^{\prime}, X\right)$.

The linear mapping in Lemma $1 \mathrm{~m}^{*}$ may not be one to one. However, we can assume that $m^{*}$ is one to one on $C(K)^{\prime \prime}[9]$. We say that $X$ is cyclic with respect to $B$ if there exists $x \in X$ such that $\overline{A(x)}=X$, where $A(x)=\{T x: T \in A\}$. Therefore, $X$ is a locally convex locally solid Riesz space [6] and as proven in [6], Corollary 4.9., $\overline{A(x)}=\operatorname{Orth}(X)$ and $Z(X)=A_{I}$, where $A_{I}$ is the order ideal generated by $I$ in $\bar{A}$. It is easily seen that if $X$ is cyclic with respect to the Boolean algebra $B$, then $X$ is an f-module over $C(K)$ [4]. Now, we can state and easily prove the following fact.

\section{Proposition 2}

Let $X$ be a quasicomplete locally convex Hausdorff space such that $\mathcal{L}(X)$ is sequentially complete and let $B \subseteq \mathcal{L}(X)$ be an equicontinuous Boolean algebra of projections, and suppose that $x \in X$ is cyclic for $B$. Then

$$
\overline{m(C(K))}=\bar{A}=\operatorname{Orth}(X),
$$

where closure is taken with respect to the strong operator topology.

The following result follows from [15].

\section{Proposition 3}

Let $X$ be a quasicomplete locally convex Hausdorff space such that $\mathcal{L}(X)$ is sequentially complete and let $B \subseteq \mathcal{L}(X)$ be an equicontinuous Boolean algebra of projections, and suppose that $x \in X$ is cyclic for $B$. Then $T \in \operatorname{Orth}(X)$ if and only if $T^{\prime} \in \operatorname{Or} t h\left(X^{\prime}\right)$. 


\section{Proof.}

It suffices to prove that if $T^{\prime} \in \operatorname{Orth}\left(X^{\prime}\right)$ then $T \in \operatorname{Orth}(X)$. By HahnBanach separation theorem, $X^{\prime}$ separates the points of $X$, hence the order dual $X^{\sim}$ of $X$ separates the points of $X$. Consider that $X$ is embedded as a Riesz subspace in $X^{\prime} \sim$. Then by [15, Theorem 142.10] the order adjoint of $T, T^{\sim}, T^{\sim} \in \operatorname{Orth}\left(X^{\prime} \sim\right) . T^{\sim}$ agrees with $T^{\prime}$ on $X$. For $x, y \in X x \wedge y=0$ implies that $T^{\sim} x \wedge y=T^{\prime} x \wedge y=0$, i. e., $T^{\prime}$ is a band preserving operator. Therefore, $T^{\prime} \in \operatorname{Orth}(X)$.

Using the continuity of $m^{*}$ and $|\sigma|\left(C(K)^{\prime \prime}, C(K)^{\prime}\right)$ - denseness of $C(K)$ in $C(K)^{\prime \prime}$ we can observe the following fact that

$$
\overline{m^{*}(C(K))}=\overline{m^{*}\left(C(K)^{\prime \prime}\right)},
$$

where closure is taken with respect to the $w^{*}$ operator topology in $\mathcal{L}\left(X^{\prime}\right)$.

\section{Proposition 4}

Let $X$ be a quasicomplete locally convex Hausdorff space such that $\mathcal{L}(X)$ is sequentially complete and let $B \subseteq \mathcal{L}(X)$ be an equicontinuous Boolean algebra of projections, and suppose that $x \in X$ is cyclic for $B$. Then

$$
\overline{m^{*}\left(C(K)^{\prime \prime}\right)}=\operatorname{Orth}\left(X^{\prime}\right),
$$

where closure is taken with respect to the $w^{*}$ operator topology in $\mathcal{L}\left(X^{\prime}\right)$.

Proof. Continuity of $m^{*}$, the preceding observation and Lemma 1 imply that

$$
\overline{m^{*}\left(C(K)^{\prime \prime}\right)} \subset \operatorname{Orth}\left(X^{\prime}\right) .
$$

Suppose that $T$ belongs to $\operatorname{Orth}\left(X^{\prime}\right)$. Then by Proposition 3 or by $[15$, Theorem 142.10] $T^{\sim} \in \operatorname{Orth}\left(X^{\prime} \sim\right)$. Since $X$ is a Ricsz subspace of $X^{\prime} \sim$ and $X^{\sim}$ separates points of $X$, the restriction of the order adjoint $T^{\sim}$ to $X$ is $T^{\prime} \in \operatorname{Orth}\left(X^{\prime}\right)$ and hence

$$
T^{\prime} \in \operatorname{Orth}(X)=\overline{m(C(K))}
$$

Then there exists a net $\left(a_{\alpha}\right)$ in $C(K)$ such that

$$
m\left(a_{\alpha}\right) \rightarrow T^{\prime} \in \mathcal{L}(X),
$$

i. e., $x^{\prime} m\left(a_{\alpha}\right) x \rightarrow x^{\prime} T^{\prime} x$ for all $x \in X, x^{\prime} \in X^{\prime}$. Now, $x^{\prime} T^{\prime} x=T x^{\prime}(x)$ and $m^{*}\left(a_{\alpha}\right)$ in $m^{*}(C(K))$ imply that $T \in \overline{m^{*}(C(K))}$.

By the preceding fact that we have $T \in \overline{m^{*}\left(C(K)^{\prime \prime}\right)}$. 
Noting that AlglatA denotes the set of all linear operators $T$ such that $T$ leaves invariant each $A$-invariant $\sigma\left(X^{\prime}, X\right)$ closed subspace of $X^{\prime}$, we can state one of the principal results of this paper.

\section{Proposition 5}

Let $B$ be an equicontinuous Boolean algebra of projections in the quasicomplete locally convex Hausdorff space $X$ such that $\mathcal{L}(X)$ is sequentially complete, and suppose that $x \in X$ is a cyclic vector for $B$. Then $m^{*}(C(K))$ is a reflexive algebra, i. e., $\overline{m^{*}(C(K))}=$ Alglat $m^{*}(C(K))$, where closure is taken with respect to the $w^{*}$ operator topology in $\mathcal{L}\left(X^{\prime}\right)$.

Proof. Let $m^{*}(C(K))=W$. It is sufficient to show that Alglat $\subseteq \subseteq \bar{W}$. Let $T \in$ Alglat $W$ and suppose that $Y$ is a band in $X^{\prime}$. For each $S \in W, S Y \subseteq Y$. Therefore, $T Y \subset Y$, i. e., $\mathrm{T}$ is a band preserving operator. Dedekind completeness of $X^{\prime}$ implies that $T \in \operatorname{Orth}\left(X^{\prime}\right)$. Hence, $T \in \bar{W}$.

The following Lemmas can be easily proven by using definitions.

\section{Lemma 6}

Let $X$ be a quasicomplete locally convex Hausdorff space such that $\mathcal{L}(X)$ is sequentially complete and let $B \subseteq \mathcal{L}(X)$ be an equicontinuous Boolean algebra of projections, and let $x, y \in X$. Then the following statements are true.

(i) A functional $x^{\prime} \in X^{\prime}$ belongs to $\overline{m(C(K))(x)}^{\circ}$ if and only if $x \cdot x^{\prime}=0$, where $\overline{m(\overline{C(K))(x)}}$ is the polar of $\overline{m(C(K))(x)}$ in $X^{\prime}$.

(ii) $y \in \Delta(x)$ if and only if $\left|y \cdot x^{\prime}\right| \leq\left|x \cdot x^{\prime}\right|$ for each $x^{\prime} \in X^{\prime}$.

Similar statements can be given in the following Lemma.

\section{Lemma 7}

Let $X$ be a quasicomplete locally convex Hausdorff space such that $\mathcal{L}(X)$ is sequentially complete and let $B \subseteq \mathcal{L}(X)$ be an equicontinuous Boolean algebra of projections, and let $x^{\prime}, y^{\prime} \in X^{\prime}$. Then the following statements are true.

(i) An clement $x \in X$ belongs to ${\overline{m^{*}\left(C\left(K^{\prime \prime}\right)\left(x^{\prime}\right)\right.}}^{o}$ if and only if $x \cdot x^{\prime}=0$, where $\overline{m^{*}\left(C(K)^{\prime \prime}\right)\left(x^{\prime}\right)}$ is the polar of $\overline{m^{*}\left(C(K)^{\prime \prime}\right)\left(x^{\prime}\right)}$ in $X$. 
(ii) $y^{\prime} \in \Delta\left(x^{\prime}\right)$ if and only if $\left|x \cdot y^{\prime}\right| \leq\left|x \cdot x^{\prime}\right|$ for all $x \in X$.

\section{Lemma 8}

Let $B$ be an equicontinuous Boolean algebra of projections in the quasicomplete locally convex Hausdorff space $X$ such that $\mathcal{L}(X)$ is sequentially complete and $T \in \mathcal{L}\left(X^{\prime}\right)$. Then the following statements are equivalent.

(i) T leaves invariant all $B$-invariant $\sigma\left(X^{\prime}, X\right)$ closed subspaces of $X^{\prime}$.

(ii) If $x \cdot x^{\prime}=0$, then $x \cdot T x^{\prime}=0$, where $x^{\prime} \in X^{\prime}, x \in X$.

Proof. It is easily seen that $X$ is a locally convex $C(K)$-module; therefore, we can prove equivalencess.

Noting that with the assumption below $m^{*}\left(C(K)^{\prime \prime}\right)$ is an order ideal in $\operatorname{Orth}\left(X^{\prime}\right)$, the following result follows from [4].

\section{Proposition 9}

Let $B$ be an equicontinuous Boolean algebra of projections in the quasicomplete locally convex Hausdorff space $X$ such that $\mathcal{L}(X)$ is sequentially complete, and suppose that $x \in X$ is a cyclic vector for $B$. Then $m^{*}(U)=Z\left(X^{\prime}\right)$, where $\mathrm{U}$ is the order ideal generated by unit element in $C(K)^{\prime \prime}$ and

$$
Z\left(X^{\prime}\right)=\left\{T \in \operatorname{Orth}\left(X^{\prime}\right): \quad|T| \leq n I \quad \text { for some } n\right\} .
$$

The one of the main results of this paper, called Dual Bade Theorem, is given by the following Theorem.

\section{Theorem 10}

Let $X$ be a quasicomplete locally convex Hausdorff space such that $\mathcal{L}(X)$ is sequentially complete, and suppose that $B$ is an equicontinuous Boolean algebra of projections in $X$. Then

$$
Z_{m}\left(X^{\prime}\right)=\operatorname{Alglatm}^{*}(C(K)) .
$$

Proof. It is sufficient to show that $\operatorname{Alglatm}^{*}(C(K)) \subseteq Z_{m}\left(X^{\prime}\right)$. Let $T \in$ Alglatm* $m^{*}(C(K))$ and fix an $x \in X$. The space $\overline{m(C(K))(x)}$ is identified with $X^{\prime} \overline{m(C(K))(x)}^{\circ}$, where $\overline{m(C(K))(x)}^{\circ}$ is the polar of $\overline{m(C(K))(x)}$ in 
$X^{\prime}$. Since $\overline{m(C(K))(x)}^{\circ}$ is $\sigma\left(X^{\prime}, X\right)$ closed and $B$-invariant subspace, $T$ leaves invariant $\overline{m(C(K))(x)}{ }^{\circ}$. Wo define a quotient map $T_{1}$ from $\overline{m(C(K))(x)}$ to $\overline{m(C(K))(x)}^{\prime}$ by means of $T_{1}\left(x^{\prime}+\overline{m(C(K))(x)}^{\circ}\right)=T x^{\prime}+\overline{m(C(K))(x)}^{\circ}$. Taking an element $x^{\prime}+\overline{m(C(K))(x)}^{\circ} \in{\overline{m\left(C\left(K^{\circ}\right)\right)(x)}}^{\prime}$ and an clement $y \in$ $\overline{m(C(K))(x)}$ such that $y \cdot\left(x^{\prime}+\overline{\left.m(C(K))(x)^{\circ}\right)}=0\right.$, we find $y \cdot x^{\prime}=0$. Hence, by Lemma $8 y \cdot T x^{\prime}=0$.It follows that $y \cdot T_{1}\left(x^{\prime}+\overline{m(C(K))(x)}^{\circ}\right)=0$. One more using Lemma 8, we sec that $T_{1} \in Z_{m}(\overline{m(C(K))(x)})$. In particular, for each $x^{\prime} \in X^{\prime}$, we have $T x^{\prime} \in \Delta\left(x^{\prime}\right)$, i. c., $T \in Z_{m}\left(X^{\prime}\right)$.

In order to prove the next result we need the following Lemma from [8].

\section{Lemma 11}

Let $X$ be a locally convex Hausdorff space, and let $D$ be a closed unital subalgebra of $\mathcal{L}(X)$, and suppose that $T \in \mathcal{L}(X)$ is given.

(i) T leaves invariant every $D$ - invariant subspace of $X$ if and only if $<$ $\left.T x, x^{\prime}\right\rangle=0$, whenerer $x \in X$ and $x^{\prime} \in X^{\prime}$ are such that $\left\langle S x, x^{\prime}\right\rangle=$ 0 for all $S \in D$.

(ii) $T \in D$ if and only if

$$
\sum_{i=1}^{n}<T x_{i}, x_{i}^{\prime}>=0
$$

whenever $x_{1}, x_{2}, \ldots, x_{n} \in X$ and $x_{1}^{\prime}, x_{2}^{\prime}, \ldots, x_{n}^{\prime} \in X^{\prime}$ are such that

$$
\sum_{i=1}^{n}<S x_{i}, x_{i}^{\prime}>=0
$$

for all $S \in D$.

Let $B_{1}$ be a Boolean algebra in $C(K)^{\prime \prime}$. Then $m^{*}\left(B_{1}\right)$ is strongly equicontinuous in $\mathcal{L}\left(X^{\prime}\right)$ [9]. As shown in [6], Proposition 3.6., the completion of lincar span of $m^{*}\left(B_{1}\right)$ called $\hat{M}$ is a Dedekind complete locally solid locally convex Ricsz space and has Lebesgue topology. Considering these results we will conclude by presenting an application of the Dual Bade Theorem.

\section{Proposition 12}

Let $X$ be a quasicomplete locally convex Hausdorff space such that $\mathcal{L}(X)$ is sequentially complete, and let $B$ be an equicontinuous Boolean algebra 
of projections in $X$. Suppose that $D$ is a closed unital subalgebra of $\hat{M}$. Then the following statements are equivalent.

(i) $T \in D$.

(ii) $T$ leaves invariant each $D$-invariant $\sigma\left(X^{\prime}, X\right)$ closed subspace of $X^{\prime}$ and $T \in \mathcal{L}\left(X^{\prime}\right)$.

Proof. It is sufficient to prove that $(i i) \Rightarrow(i)$. Suppose that $T$ leaves invariant $D$-invariant $\sigma\left(X^{\prime}, X\right)$ closed subspace of $X^{\prime}$ and $T \in \mathcal{L}\left(X^{\prime}\right)$. It is obvious that $T \in Z_{m}\left(X^{\prime}\right)$ by Theorem 10. By Lemma 11, we have the following: $T \in D \Longleftrightarrow$

$$
\sum_{i=1}^{n}<x_{i}, T x_{i}^{\prime}>=0,
$$

whenever

$$
\sum_{i=1}^{n}<x_{i}, S x_{i}^{\prime}>=0
$$

for $x_{1}, x_{2}, \ldots, x_{n} \in X, x_{1}^{\prime}, x_{2}^{\prime}, \ldots, x_{n}^{\prime} \in X^{\prime}$ and all $S \in D$. As slown in [8], Proposition 3.2., there exist $x^{\prime} \in X^{\prime}, x \in X$ such that $\left\langle x, S x^{\prime}\right\rangle=0$ for all $S \in D$. Let $Y$ be a $\sigma\left(X^{\prime}, X\right)$ closed subspace of $X^{\prime}$ generated by $\left\{S x^{\prime}: S \in D\right\}$. Clearly $S Y \subseteq Y$, and therefore $T Y \subseteq Y$. Obviously the clement $x^{\prime} \in Y$. It implies that $x \perp Y$, i. c., $\langle x, y\rangle=0$ for all $y \in Y$. Wo conclude that $\left\langle x, T x^{\prime}\right\rangle=0$. By Lemma 11, wo have $T \in D$.

\section{References}

[1] Abramovich Y. A., Arenson E. L. and Kitover A. K., Banach $C(K)$ modules and operators preserving disjointmess, Pit. Res. Not. in Math.Ser., England (1992).

[2] Aliprantis C. D. and Burkinshaw O., Locally solid Ries\% spaces, New York-San Francisco-London, Academic Press (1978).

[3] Aliprantis C. D. and Burkinshaw O., Positive Operators, New YorkSan Francisco London, Academic Press (1985).

[4] Alpay S.. and Turan B., On f-modules, preprint.

[5] Bade W.G., On Boolean algebras of projections and algebras of operators, Trans.Amer.Math. Soc. 80, pp. $345-359$ (1955). 
[6] Dodds P. G. and Pagter de B., Orthomorphism and Boolean algebras of projections, Math.Z.187, pp. $361-381$ (1984).

[7] Dodds P. G. and Ricker W.J., Spectral measures and the Bade reflexivity theorem, J. Funct. Anal. 61, pp. 136 - 163, (1985).

[8] Dodds P. G., Ricker W. J. and Pagter de B., Reflexivity and order properties of scalar-type spectral operators in locally convex spaces. Trans. Amer. Math. Soc. 293, pp. 355 - 380, (1986).

[9] Gök Ö., On a Bade type reflexive algebra, Ph. D. Thesis, METU, Ankara (1990).

[10] Gillespie T. A., Boolean algebras of projections and reflexive algebras of operators, Proc.London Math. Soc. 37, pp. 56 - 74, (1978).

[11] Hadwin D. and Orhon M., Reflexivity and Approximate Reflexivity for Bounded Boolean Algebras of Projections, J. Funct.Anal.87, pp. $348-358$, (1989).

[12] Rall C., Über Booleshe Algebren von Projektionen, Math.Z.153, pp. $199-217,(1977)$.

[13] Ricker W. J., On Boolcan algcbras of projections and scalar-type spectral operators, Proc. Amer. Matl. Soc. 87, pp. 73 - 77, (1983).

[14] Ricker W. J., Uniformly closed algebras generated by Boolean algebras of projections in locally convex spaces, Canad. J. Math.39, pp. $1123-1146,(1987)$.

[15] Zaanen A. C., Riesz Spaces II, Amsterdam-New York-Oxford, (1983).

Received : July 10, 1998.

\section{Ömer Gök}

Department of Nathematics

Faculty of Arts and Sciences

Yildiz Teclnical University

80270 Sisli

Istanbul - TURKEY 